\title{
Economicidade da idade de reforma de canaviais
}

Economicity of the reform age of sugarcane fields

\author{
Júnio Gomes*; Miklos Maximiliano Bajay²
}

1* ESALQ/USP - Engenheiro agrônomo - Av. Centenário, 303 - São Dimas - CEP 13416-000 - Piracicaba (SP), Brasil <eng.juniogomes@gmail.com>

${ }^{2}$ ESALQ/USP - Doutor em Genética e Melhoramento de Plantas - Avenida Centenário, 303 - São Dimas - CEP 13416-000 - Piracicaba (SP), Brasil

\section{Resumo}

A reforma de canaviais é, atualmente, uma decisão, fundamentalmente técnica, calcada na experiência de técnicos, sobretudo, no uso de importantes indicadores subsidiadores dessa tomada de decisão, para fins de obtenção de lucro e, portanto, é fundamental o uso de instrumentos de análise que possam auxiliar na gestão de projetos. Este trabalho teve por objetivo aplicar o uso de indicadores de análise econômica de projetos, avaliando os retornos financeiros de um ciclo do canavial, em diferentes estágios de corte, visando determinar o estágio de corte que oferecesse o ponto econômico mais adequado para a sua reforma e a possibilidade de indicar o seu uso como opção rápida, segura e prática na decisão de reforma do canavial. Alguns dados, indicadores de produção, definidos como premissas básicas, serviram para os cálculos do Valor Presente Líquido com datas diferentes [XVPL] e do Valor Presente Líquido Anual [VPLA] para um canavial da região tradicional do Centro-Sul do Brasil. O VPLA foi utilizado, por ser um método que compara projetos com períodos diferentes o que o fez relevante para solução do problema proposto neste trabalho. Após análise, sem variar os indicadores, verificou-se que a reforma do canavial pode ser feita no sétimo corte. Quando foi estudada a variação de diferentes indicadores de produção e cenários, em análise de sensibilidade, houve alternância do ponto econômico ideal para reforma, indicando, portanto, mudança do estágio de corte ideal de reforma, isso indica que a ferramenta é prática, flexível, podendo auxiliar e, ser indicada na tomada de decisão de reforma de canaviais pelos produtores e gestores do setor.

Palavras-chave: análise econômica, cana de açúcar, estágio de corte, indicadores

\begin{abstract}
The sugarcane reformation is a technical decision based in the experience of technicians, above all, in the use of important subsidiaries indicators of this decision-making for purposes of profit making and, therefore the use of instruments of analysis that can help in the management of the projects is fundamental. The objective of this work was to apply the indicators of economic analysis of projects, evaluating the financial returns of a sugarcane cycle, in different cutting stages, in order to determine the cutting stage that offered the most appropriate economic point for its reform and the possibility of indicating its use as a fast, safe and practical option in the sugarcane reforestation decision.Production indicators were used to calculate the Net Present Value with different dates [XNPV] and the Annualized Net Present Value [ANPV] for a sugarcane field in the traditional region of Central-South Brazil. The ANPV was used, because it is a method that compares projects with different periods which made it relevant to solve the problem proposed in this work. After analysis, without changing the indicators, it was verified that the sugarcane reformation can be done in the seventh cut. When the variation of different production indicators and scenarios was studied, in the sensitivity analysis, there was alternation of the ideal economic point to reform, indicating, therefore, a change of the ideal cutting stage of reform, this indicates that the tool is practical, flexible and can help, and be indicated in the decision making of sugarcane fields reformation by the producers and managers of the sector.
\end{abstract}

Keywords: Economic analysis, sugarcane, cutting stage, indicators

\section{Introdução}

A cana-de-açúcar apresenta ciclos vegetativos médios de cinco safras e tem por característica a intensa mecanização para a manutenção da cultura desde a sua fundação e tratos culturais até a colheita (Cruz e Magalhães, 2013). A longevidade do canavial depende de vários fatores, tais como: de suas características biológicas, dos tratos culturais e o tipo de manejo adotado, das condições edafoclimáticas do local onde está implantado, e os seus reflexos estão na produtividade da lavoura que é um indicador muito usado para definir o momento da reforma. A pesquisa varietal tem contribuído para ganhos de produtividade ao longo do ciclo produtivo da cana-de-açúcar, oferecendo variedades com potencial para vários cortes (Borba e Bazzo, 2009). O canavial pode apresentar longevidade reduzida, quando a colheita é mecanizada. Tal prática influencia na rebrota, tornando-a irregular ou deficiente, 
em função do esmagamento e maceração de colmos, da altura inadequada de corte, da remoção de soqueiras e da compactação (Mello, 2005).

Após cada corte o canavial vai se exaurindo com redução gradativa da produtividade e das respostas aos tratos culturais da soqueira sendo necessária a reforma do canavial para que haja a manutenção do fluxo contínuo de matéria-prima à indústria sucroenergética e a continuidade do ciclo de produção do açúcar e álcool.

A implantação de um novo canavial necessita de alto investimento e a busca por canaviais com longevidade alta é o desafio de todos os profissionais. A reforma do canavial é uma atividade complexa, porém necessária para viabilidade econômica da empresa e exige um planejamento técnico, operacional e financeiro que atenda a necessidade de formação de viveiros para mudas, alocação de novas variedades nos diferentes ambientes de produção, distância da unidade industrial e volume de matéria-prima para atender a demanda industrial (Borba e Bazzo, 2009).

$\mathrm{Na}$ prática, a decisão da reforma do canavial é balizada por monitoramento da produtividade obtida na ocasião de cada colheita, aliado a avaliações das condições da lavoura por técnicos com experiência no setor (Borba e Bazzo, 2009). A decisão de reformar ou não um canavial pode ser feita pelo uso da contabilidade agrícola e de estimativas de custos que tem por finalidade oferecer instrumentos racionais de decisão, através de indicadores gerenciais construídos a partir dessa base de dados (Veiga Filho, 2002).

Para tal decisão, torna-se importante fazer uma análise criteriosa do investimento, baseando-se em técnicas que favorecem a comparação de resultados entre projetos de forma científica (Kuhnen e Bauer, 1996). A sobrevivência de uma empresa está baseada nas decisões de capital, e são cruciais, pois podem tanto consolidar uma trajetória de expansão, quando oportunas e no tempo certo, quanto comprometer a sua própria sobrevivência (Souza e Clemente, 2004). É fundamental reduzir os riscos na tomada de decisão e isso é possível a partir das análises e avaliações relativas às decisões de capital, com diminuição do nível de incertezas pelo uso de ferramentas de análise de investimentos de fácil acesso, porém pouco usadas no setor sucroalcooleiro.

O objetivo deste trabalho teve por base o conceito de que o melhor resultado de VPLA atingido em um ciclo produtivo do canavial é quem define o melhor ponto econômico do projeto, sendo, portanto, um parâmetro orientador, assertivo e facilitador do momento ideal de reforma da lavoura aos agentes tomadores de decisão do setor sucroenergético, portanto, utilizou-se a ferramenta de administração rural, Valor Presente Líquido Anualizado [VPLA] sobre os resultados dos fluxos de caixa livre, determinados para diferentes ciclos de canaviais, tendo-se como base, alguns dados considerados importantes indicadores de produção disponíveis (PECEGE, 2015).

\section{Material e Métodos}

\section{Fontes de dados}

O trabalho em questão tem uma abordagem quantitativa $\mathrm{e}$ teve seus dados obtidos conforme descrição a seguir:

Os indicadores: valor dos desembolsos para a formação do canavial, das operações de tratos culturais de cana soca, o valor do Açúcar Total Recuperável [ATR] da cana para fins de cálculo de receita no Fluxo de Caixa Livre [FCL], o ATR padrão, o preço do ATR padrão e a taxa de arrendamento para fins de cálculo do pagamento de arrendamento da terra referem-se à safra 2014/2015 e foram obtidos em PECEGE (2015). O ATR da cana $\left(\mathrm{kg} \mathrm{t}^{-1}\right)$ usado no trabalho foi obtido pela média da soma dos ATRs (cana processada + cana fornecedor), expressos em $\mathrm{kg} \mathrm{t}^{-1} \mathrm{e}$, o valor de desembolsos para a Formação do canavial foi calculado multiplicando-se o valor da depreciação da formação do canavial $\left(\mathrm{R} \$ h^{-1}\right)$ por cinco cortes, que foi o número de cortes antes do replantio do canavial adotado em PECEGE (2015).

Para a realização desse trabalho foi considerado um canavial de ano e meio, com produtividade inicial no $1^{\circ}$ corte de $114 \mathrm{tha}^{-1}$, com quebra de $12 \%$ de produtividade do $1^{\circ}$ para o $2^{\circ}$ corte e uma média constante de redução de $8 \%$ para os demais cortes, coletados em histórico de uma usina da região de Araçatuba, estado de São Paulo, e utilizada uma taxa de juros real de 5,09\% ao ano [a.a.], que é resultado da taxa de juros do sistema especial de liquidação e custódia [SELIC] de 14\% com a aplicação da taxa de juros projetada de $8,48 \%$ a.a., conforme dados do Banco Central do Brasil (BACEN, 2016).

Os serviços de colheita foram tratados como sendo terceirizados e, portanto, foram excluídas as depreciações de maquinários envolvidos nessas operações. Considerou-se o fato de que depreciação e amortização são consideradas como itens não relevantes, que servem apenas para o cálculo do imposto de renda (IR) e que não interferem no resultado do fluxo de caixa. Assaf Neto (1992) cita que nestes fluxos são computados somente os movimentos efetivos de recursos com reflexos financeiros sobre o caixa, desprezando-se receitas e despesas de natureza eminentemente contábil (depreciação, amortização, reavaliação patrimonial, entre outros resultados que não são pagos ou recebidos em termo de caixa).

\section{Método para análise dos dados}

A avaliação de investimento foi executada a partir de fluxo de caixa livre, medido para cada período do intervalo de tempo, considerando os ciclos de cinco, seis, sete e oito anos. Cada ciclo foi analisado através do uso dos indicadores econômicos de viabilidade financeira, a saber: Valor Presente Líquido para períodos não uniformes [XVPL] e VPLA.

Justifica-se o uso do XVPL porque a análise de resultado do $1^{\circ}$ corte da cana de açúcar, para canaviais de ano e meio, abrange o período de dezoito meses, sendo 
nos demais cortes um período de doze meses. Para este trabalho foram estabelecidas as datas de 15 mar. 2016, como data da formação da lavoura, com colheita em 15 ago. 2017 e, a partir daí, sucessivas colheitas nos meses de agosto, ano após ano até o $8^{\circ}$ corte.

A análise de VPLA, em cada ciclo do canavial, foi feita para três situações diferentes. $\mathrm{Na}$ primeira, apenas os dados originais, estabelecidos no trabalho, serviram de referência para a análise, resultando em um VPLA para o projeto, sem análise de sensibilidade. $\mathrm{Na}$ segunda situação, foram analisados os principais indicadores de produção que afetam o Fluxo de Caixa Livre dos projetos, por meio de sensibilidade, considerando as suas variações. Por fim, a terceira situação onde foram analisados os efeitos das interações, aos pares, desses indicadores de produção, também por meio de análise de sensibilidade, nos resultados econômicos.

\section{Instrumentos de análise de investimentos}

Diversas são as ferramentas de análise, como opção para cada tipo de investimento, seja ele de grande ou de pequeno porte, demandando horizontes de planejamento maiores ou menores (Souza e Clemente, 2004). Os principais indicadores de análise de investimentos são divididos em dois grupos. Um deles refere-se a indicadores associados à rentabilidade (ganho ou criação de riqueza) do projeto, são eles: Valor Presente Líquido [VPL], Valor Presente Líquido Anualizado, Índice Benefício/Custo [IBC], Retorno Adicional sobre o Investimento [ROIA], Taxa Interna de Retorno [TIR]. No outro grupo estão os indicadores associados ao risco do projeto, dentre eles estão: Período de Recuperação do Investimento (Payback), Custo de Oportunidade [CO], Ponto de Fischer como medida de risco, Retorno sobre o Patrimônio Líquido [RSPL], Índice de Lucratividade [IL] e também a Taxa Interna de Retorno. Foi calculado o VPLA dos fluxos de caixa livre nesse trabalho por se tratar de análise de fluxos de caixa de projetos com vida útil diferentes, sendo, portanto, essa a ferramenta indicada para o caso.

\section{Valor Presente Líquido [VPL]}

O valor presente líquido é um método simples e prático de ser utilizado e por isso é bastante comum o seu uso nas análises de investimentos para tomadas de decisão. É um método que se baseia em analisar projetos onde o investimento tenha maiores resultados para os investidores do que o seu custo, sendo assim, valores positivos de VPL em uma análise de investimento tornam o projeto atrativo e viável (Samanez, 2001).

Pode ser definido como a soma algébrica dos valores descontados do fluxo de caixa a ele associado, bem como a diferença, em valores absolutos do investimento, entre os valores presentes das entradas e das saídas de caixa (Assaf Neto, 1992) Quando se tem períodos diferentes no Fluxo de Caixa, adota-se o XVPL que traz ao valor presente todos os valores, considerando os prazos diferentes de colheita, principalmente no caso de canaviais de ano e meio, onde no primeiro corte da cana de açúcar leva, em média, 18 meses entre a formação da lavoura e a colheita e depois, nos demais cortes, em torno de 12 meses.

Dá-se o nome de vida econômica ao intervalo entre a data de investimento inicial e a data de retorno do capital e, a análise da vida econômica de projetos deve levar em consideração duas situações: casos onde o investimento envolve vidas econômicas iguais e, casos com vidas econômicas diferentes (Marim, 1980).

Algumas vezes, porém, principalmente para projetos com horizontes de planejamento longos, o desempenho do valor monetário do VPL apresenta dificuldade para comparação e como alternativa surge o VPLA.

\section{Valor Presente Líquido Anualizado [VPLA]}

O VPLA é um método que uniformiza o fluxo de caixa correspondente, ano a ano, da vida econômica do projeto como se fosse uma média para cada projeto.

Ele permite a comparação mesmo para projetos com horizontes de planejamentos distintos (Souza e Clemente, 2004). Os valores deverão ser crescentes ao longo do tempo até atingir um máximo, tornando-se depois decrescentes. O valor máximo estará representando o número ótimo de cortes (Veiga Filho, 2002).

É mais fácil para o tomador de decisão raciocinar em termos de ganho por período, do que em termos de ganho acumulado ao longo de diversos períodos. A fórmula para o cálculo do VPLA é apresentada pela eq. (1), Souza (2004).

$$
\text { VPLA }=V P L *\left(\frac{i^{*}(1+i)^{\mathrm{n}}}{(1+\mathrm{i})^{\mathrm{n}}-1}\right)
$$

onde, VPL: é o Valor Presente Líquido; i: Taxa de juros; n: Horizonte de tempo.

$$
\begin{aligned}
& \text { OVPL foi calculado pela eq. (2): } \\
& \qquad V P L=\sum_{j=1}^{n} \frac{F C j *(1+i)^{n-j}}{(1+i)^{n}}-F C o
\end{aligned}
$$

onde, FCj: Fluxo de caixa no período j, com j variando de 1 a n; i: Taxa de juros; n: período e FCo: Fluxo de caixa no início.

\section{Análise de sensibilidade}

A análise de sensibilidade é uma estratégia utilizada para simulações de cenários onde é possível avaliar, isoladamente, os possíveis impactos da alteração de alguns indicadores de produção e até mesmo a interação desses indicadores, analisados conjuntamente, nos resultados que estão sendo analisados. Os indicadores de produção estudados na análise de sensibilidade, isoladamente, foram: produtividade agrícola $\left(\mathrm{t} \mathrm{ha}^{-1}\right)$, taxa real de juros (\%), preço da tonelada de cana recebida ( $\mathrm{R} \$$ $\left.\mathrm{t}^{-1}\right)$, taxa de arrendamento $\left(\mathrm{R} \$ \mathrm{ha}^{-1}\right)$, custo desembolsável da colheita $\left(R \$ t^{-1}\right)$, custo desembolsável da formação da 
lavoura $\left(\mathrm{R} \$ \mathrm{ha}^{-1}\right)$ e custo desembolsável dos tratos culturais da cana-soca $\left(\mathrm{R} \$ \mathrm{ha}^{-1}\right)$.

As interações entre os indicadores de produção nos resultados do fluxo de caixa livre impactando conjuntamente os resultados de VPLA estudados foram: taxa real de juros (\%) versus preço da tonelada de cana, custo dos tratos culturais da cana-soca versus produtividade agrícola, custo desembolsável da formação da lavoura versus produtividade agrícola e preço da cana versus produtividade agrícola.

São descritos a seguir esses indicadores de produção e suas justificativas para adoção no estudo de seus impactos no VPLA, calculados por meio do teste de hipótese, variando os indicadores de interesse e mantendo-se os demais dados dos fluxos de caixa livre analisados no trabalho.

\section{Indicadores de produção analisados \\ Produtividade agrícola}

A produtividade resulta da produção obtida em uma unidade de área, representada por $\mathrm{t} \mathrm{ha} \mathrm{h}^{-1}$. Sofre grande influência de fatores climáticos e de manejo.

Estratégias de manejo com alterações no uso de insumos adotados na formação do canavial e nos tratos culturais da soqueira impactam nos custos de produção, mas geralmente tem impacto direto na produtividade.

\section{Taxa real de juros}

Na seleção de investimentos é necessária a definição prévia da taxa de juros. É um indicador técnico que impacta muito nos custos das operações, sendo assim, tem importância na análise de simulações, pois o projeto deve ter remuneração superior àquelas taxas disponíveis no mercado.

A eq.(3) apresenta como foi calculada a taxa de juros nesse trabalho.

$$
\mathrm{i}(\mathrm{real})=\left(\frac{1+\mathrm{i}(\text { nominal })}{1+\mathrm{i}(\text { inflação })}\right)-1
$$

onde $i$ (nominal): é a taxa SELIC; $i$ (inflação): é a taxa de inflação projetada no ano.

\section{Preço da tonelada da cana}

O preço da cana é calculado multiplicando-se o preço do $\mathrm{kg}$ de ATR, que é calculado pelo Conselho de Produtores de Cana-de-açúcar, Açúcar e Etanol do estado de São Paulo [CONSECANA] e a quantidade de ATR médio da cana processada (PECEGE, 2015). Os preços do kg de ATR baseados no CONSECANA é uma variável exógena, sendo assim, não há possibilidade de adotar estratégias que possam alterá-lo. A única alternativa é trabalhar com as projeções possíveis para o preço da cana, em $\mathrm{R} \$ \mathrm{t}^{-1}$, baseando-se em históricos e expectativas de mercado, respaldados pelas principais instituições responsáveis por esses dados no setor sucroenergético.

\section{Taxa de arrendamento}

É um indicador diretamente ligado à região onde está inserida a empresa e requer uma eficiente estratégia administrativa nas negociações de arrendamento pois tem papel fundamental neste indicador que sofre muita concorrência com as outras atividades de uma determinada região. Dessa forma, os valores podem variar muito e em alguns casos até inviabilizar alguns projetos. Além disso, a taxa de arrendamento é muito influenciada pelo preço histórico da terra, pela fertilidade do solo, além da topografia, distância da unidade industrial, acessibilidade, tamanho da área entre outras características.

\section{Custo desembolsável da colheita}

A atividade de colheita do canavial envolve muitos gastos. É necessário um alto investimento em máquinas e equipamentos por isso muitos produtores optam por terceirizar essa etapa do processo produtivo.

Considerando-se que é um item do custo que pode ser negociado no momento de sua contratação, foi estudado neste trabalho como opção de análise de suas variações nos resultados do Fluxo de Caixa do projeto.

\section{Custo desembolsável da formação da lavoura}

Este indicador tem um forte impacto no Fluxo de Caixa, sendo necessário muito critério no momento dos desembolsos para essa etapa da lavoura.

É uma fase da lavoura onde ocorrem desembolsos em proporções elevadas, por isso qualquer ação gerencial provoca interferências acentuadas nos resultados econômicos do projeto.

O sucesso e a longevidade da lavoura dependem muito de uma boa formação do canavial, portanto é importante que se pense em redução dos custos operacionais nesta etapa não deixando de pensar na qualidade do plantio.

\section{Custo desembolsável de tratos culturais da cana soca}

Esse indicador tem grande influência nos resultados da produtividade dos canaviais. Baixos investimentos em tratos de cana soca podem ocasionar quebras na produtividade, portanto variações neste indicador podem acarretar em redução na produtividade dos canaviais. Pode-se trabalhar esse custo com a adoção de medidas racionais de manejo que busquem o aumento de produtividade com baixos custos de investimentos. É um indicador fortemente influenciado pelo momento econômico do setor.

\section{Interação entre os indicadores de produção}

Alguns indicadores quando submetidos a variações podem, por consequência, provocar influências em outros, portanto algumas dessas interações foram estudadas neste trabalho com a finalidade de verificar os seus efeitos nos resultados econômicos. 
Interação entre Taxa real de juros e Preço da cana

O impacto da variação da taxa de juros tem reflexo nos custos operacionais e a análise da variação do preço da cana tem a sua importância porque ele é quem determina a receita no projeto.

Interação entre Custo desembolsável dos Tratos de Cana-soca e Produtividade agrícola

Dependendo da situação econômica da empresa, pode ser necessária a redução de insumos e de algumas despesas operacionais nos tratos da soqueira, entretanto essa redução dos custos dos tratos da cana-soca poderá refletir na produtividade do canavial.

Interação entre Custo desembolsável para a formação da Lavoura e Produtividade agrícola

O custo de implantação de uma lavoura de cana-deaçúcar é alto e requer grande desembolso e a produtividade tem grande impacto na decisão de reformar ou não o canavial.

\section{Interação entre Preço da Cana e Produtividade agrícola}

Em geral, as condições de preço da cana têm impacto direto sobre a geração de renda e, portanto, na tomada de decisão de reforma de canaviais. A produtividade recebe a influência do preço da cana nos momentos de decisões de investimentos com insumos e operações. De certa forma, quanto menor o preço da cana, menores são os investimentos aplicados na cultura e quanto maior o preço, melhores e maiores são os recursos disponibilizados para os canaviais.

\section{Resultados e Discussão}

Adotou-se que a empresa estudada é uma tomadora de serviços, portanto foram descartadas as depreciações para as diversas operações. As Tabelas 1, 2 e 3 apresentam, respectivamente, os dados dos valores adotados de desembolso na formação do canavial, o custo desembolsável para as operações de tratos culturais da cana-soca, e o valor desembolsável para a operação de corte, carregamento e transporte de cana própria por estágio e por item de custo para a região Centro-Sul tradicional, na safra 2014/2015, extraídos de PECEGE (2015) com adaptações. A análise considerou os dados referentes à unidade padrão de 1 ha para facilitar a comparação e a praticidade do estudo a que se propôs o trabalho.

Os indicadores de produção utilizados no Fluxo de Caixa Livre estão citados como premissas básicas e apresentados na Tabela 4.
Foi utilizada a taxa real de juros, calculada com base na taxa básica da economia brasileira, a taxa do sistema especial de liquidação e custódia [SELIC], obtida junto ao Banco Central do Brasil (BACEN, 2016) no mês de outubro de 2016 , com valor de $14 \%$ e com base na taxa de inflação do índice de preços do consumidor ao atacado [IPCA], período de 12 meses, também obtida na mesma fonte e projetada em 8,48\% a.a.

Tabela 1. Desembolsos para formação da lavoura, para a região Centro-Sul tradicional

\begin{tabular}{ll}
\hline \multicolumn{1}{c}{ Item } & Custo \\
\hline & ------- \\
Mecanização & 3.125 \\
Mão-de-obra & 1.165 \\
Insumos & 2.305 \\
\hline Desembolso & 6.595 \\
\hline
\end{tabular}

Fonte: adaptado de PECEGE (2015)

Tabela 2. Custos desembolsáveis de tratos culturais de cana-soca, para a região Centro-Sul tradicional

\begin{tabular}{lc}
\hline \multicolumn{1}{c}{ Item } & Custo \\
\hline & ------- \\
Mecanização & 373 \\
Mão-de-obra & 193 \\
Insumos & 515 \\
\hline Desembolso & 1.081 \\
\hline
\end{tabular}

Fonte: adaptado de PECEGE (2015)

Tabela 3. Custos desembolsáveis de colheita de cana-deaçúcar, para a região Centro-Sul tradicional

\begin{tabular}{lc}
\hline \multicolumn{1}{c}{ Item } & Custo \\
\hline & $------\mathrm{R} \$ \mathrm{t}^{-1}----$ \\
Mecanização & 22,00 \\
Mão-de-obra & 3,56 \\
\hline Desembolso & 25,56 \\
\hline
\end{tabular}

Fonte: adaptado de PECEGE (2015)

Foram calculados os Fluxos de Caixa Livre para os diferentes ciclos da cultura considerando-se desde a sua formação até o $5^{\circ}$ corte, até o $6^{\circ}$ corte, até o $7^{\circ}$ corte e até o $8^{\circ}$ corte, a partir dos dados estabelecidos na tabela 4 e os seus resultados estão apresentados nas tabelas 5, 6,7 e 8 , respectivamente, e representam o resultado dos recebimentos menos os desembolsos com a formação da lavoura, colheita (corte, carregamento e transporte), tratos culturais de cana-soca, desembolsos com arrendamento e Fundo de Assistência ao Trabalhador Rural [FUNRURAL]. 
Tabela 4. Indicadores de Produção (Premissas básicas) utilizados no estudo econômico, para os diferentes ciclos da cultura, com as suas unidades e seus respectivos valores atribuídos

\begin{tabular}{lcc}
\hline \multicolumn{1}{c}{ Indicador de produção } & Unidade & Valor atribuído \\
\hline Desembolso formação da lavoura & 6.595 \\
Custo desembolsável da colheita & $\mathrm{R} \mathrm{ha}^{-1}$ & 25,56 \\
Custo desembolsável tratamento cana-soca & ${\mathrm{R} \$ \mathrm{t}^{-1}}^{-1}$ & 1.081 \\
Taxa de arrendamento & $\mathrm{R} \mathrm{ha}^{-1}$ & 18 \\
Preço do ATR & $\mathrm{R} \mathrm{ha}^{-1}$ & 0,4763 \\
ATR do arrendamento & $\mathrm{R} \mathrm{kg}^{-1}$ & 121,97 \\
Desembolso do arrendamento (anual) & $\mathrm{kg} \mathrm{kg}^{-1}$ & 1.047 \\
Teor do ATR da cana & $\mathrm{kg} \mathrm{t}^{-1}$ & 136,30 \\
Preço da cana & $\mathrm{R}^{-1}$ & 64,92 \\
FUNRURAL & $\%$ & 2,3 \\
Taxa real de juros anual & $\%$ & 5,09 \\
Produtividade do $1^{\circ}$ corte & $\mathrm{t} \mathrm{ha}^{-1}$ & 114 \\
Perda de produtividade no $1^{\circ}$ corte & $\%$ & 12 \\
Perda de produtividade para demais cortes & $\%$ & 8 \\
\hline
\end{tabular}

Fonte: adaptado de PECEGE (2015) e BACEN (2016)

Tabela 5. Fluxo de Caixa livre [FCL] para reforma de canavial com cinco cortes

\begin{tabular}{|c|c|c|c|c|c|}
\hline Estágio da cultura & Produtividade & Desembolso* & Recebimentos & FUNRURAL** & FCL \\
\hline & 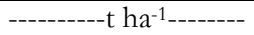 & - & - & -------- & \\
\hline Formação & - & 6.595 & 0 & 0 & -6.595 \\
\hline $1^{\circ}$ corte & 114 & 5.042 & 7.401 & 170 & 2.189 \\
\hline $2^{\circ}$ corte & 100 & 4.692 & 6.513 & 150 & 1.671 \\
\hline $3^{\circ}$ corte & 92 & 4.487 & 5.992 & 138 & 1.367 \\
\hline $4^{\circ}$ corte & 85 & 4.298 & 5.512 & 127 & 1.087 \\
\hline $5^{\circ}$ corte & 78 & 3.044 & 5.071 & 117 & 1.910 \\
\hline
\end{tabular}

* desembolsos com formação da lavoura, colheita, tratos culturais de cana-soca e arrendamento; ** Fundo de Assistência ao

Trabalhador Rural

Fonte: adaptado de PECEGE (2015)

Tabela 6. Fluxo de Caixa livre [FCL] para reforma de canavial com seis cortes

\begin{tabular}{|c|c|c|c|c|c|}
\hline Estágio da cultura & Produtividade & Desembolso* & Recebimentos & FUNRURAL** & FCL \\
\hline & ---------t ha-1-------- & ------R\$ ha-1------ & ------------- & - & \\
\hline Formação & - & 6.595 & 0 & 0 & -6.595 \\
\hline $1^{\circ}$ corte & 114 & 5.042 & 7.401 & 170 & 2.189 \\
\hline $2^{\circ}$ corte & 100 & 4.692 & 6.513 & 150 & 1.671 \\
\hline $3^{\circ}$ corte & 92 & 4.487 & 5.992 & 138 & 1.367 \\
\hline $4^{\circ}$ corte & 85 & 4.298 & 5.512 & 127 & 1.087 \\
\hline $5^{\circ}$ corte & 78 & 4.124 & 5.071 & 117 & 830 \\
\hline $6^{\circ}$ corte & 72 & 2.884 & 4.666 & 107 & 1.675 \\
\hline
\end{tabular}

* desembolsos com formação da lavoura, colheita, tratos culturais de cana-soca e arrendamento; ** Fundo de Assistência ao

Trabalhador Rural

Fonte: adaptado de PECEGE (2015)

Tabela 7. Fluxo de Caixa Livre [FCL] para reforma de canavial com sete cortes

\begin{tabular}{|c|c|c|c|c|c|}
\hline Estágio da cultura & Produtividade & Desembolso* & Recebimentos & FUNRURAL** & FCL \\
\hline & ----------t ha'-1------- & ------R\$ ha-1----- & 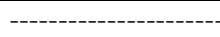 & 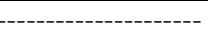 & \\
\hline Formação & - & 6.595 & 0 & 0 & -6.595 \\
\hline $1^{\circ}$ corte & 114 & 5.042 & 7.401 & 170 & 2.189 \\
\hline $2^{\circ}$ corte & 100 & 4.692 & 6.513 & 150 & 1.671 \\
\hline $3^{\circ}$ corte & 92 & 4.487 & 5.992 & 138 & 1.367 \\
\hline $4^{\circ}$ corte & 85 & 4.298 & 5.512 & 127 & 1.087 \\
\hline $5^{\circ}$ corte & 78 & 4.124 & 5.071 & 117 & 830 \\
\hline $6^{\circ}$ corte & 72 & 3.965 & 4.666 & 107 & 594 \\
\hline $7^{\circ}$ corte & 66 & 2.737 & 4.292 & 99 & 1.456 \\
\hline
\end{tabular}

* desembolsos com formação da lavoura, colheita, tratos culturais de cana-soca e arrendamento; ** Fundo de Assistência ao

Trabalhador Rural

Fonte: adaptado de PECEGE (2015) 
Tabela 8. Fluxo de Caixa Livre [FCL] para reforma de canavial com oito cortes

\begin{tabular}{|c|c|c|c|c|c|}
\hline Estágio da cultura & Produtividade & Desembolso* & Recebimentos & FUNRURAL** & FCL \\
\hline & $\mathrm{t} \mathrm{ha}^{-1}$ & $\mathrm{R} \$ \mathrm{ha}^{-1}$ & \multicolumn{2}{|c|}{--- } & \\
\hline Formação & - & 6.595 & 0 & 0 & -6.595 \\
\hline $1^{\circ}$ corte & 114 & 5.042 & 7.401 & 170 & 2.189 \\
\hline $2^{\circ}$ corte & 100 & 4.692 & 6.513 & 150 & 1.671 \\
\hline $3^{\circ}$ corte & 92 & 4.487 & 5.992 & 138 & 1.367 \\
\hline $4^{\circ}$ corte & 85 & 4.298 & 5.512 & 127 & 1.087 \\
\hline $5^{\circ}$ corte & 78 & 4.125 & 5.071 & 117 & 830 \\
\hline $6^{\circ}$ corte & 72 & 3.965 & 4.666 & 107 & 594 \\
\hline $7^{\circ}$ corte & 66 & 3.818 & 4.292 & 99 & 375 \\
\hline $8^{\circ}$ corte & 61 & 2.602 & 3.949 & 91 & 1.256 \\
\hline
\end{tabular}

* desembolsos com formação da lavoura, colheita, tratos culturais de cana-soca e arrendamento; ** Fundo de Assistência ao Trabalhador Rural

Fonte: adaptado de PECEGE (2015)

A tabela 9 apresenta as datas estabelecidas pelo autor, como referência, para cálculo do XVPL, conforme as ocorrências em cada estágio da cultura. A escolha das datas foi definida meramente para facilitar a exemplificação do uso do indicador econômico.

Considerou-se, que o $1^{\circ}$ corte foi realizado 18 meses após o seu plantio, e, que os demais cortes foram realizados após 12 meses da última colheita, por essa razão foi usado o XVPL que é indicado para situações de projetos com períodos não homogêneos.

Caso os períodos fossem homogêneos, o VPL poderia ter sido utilizado ao invés do XVPL.

A partir dos resultados dos FCL para os estágios da cultura de $5^{\circ}$ ao $8^{\circ}$ corte, foram encontrados os resultados dos indicadores econômicos XVPL e VPLA, com base na taxa real de juros real de 5,09\% a.a. e estão apresentados na Tabela 10
Tabela 9. Demonstração de datas de ocorrência para cada estágio da cultura para fins de cálculo do Valor Presente Líquido para períodos não uniformes [XVPL]

\begin{tabular}{cc}
\hline Estágio da cultura & Data da ocorrência \\
\hline Formação & 15 mar. 2016 \\
$1^{\circ}$ corte & 15 ago. 2017 \\
$2^{\circ}$ corte & 15 ago. 2018 \\
$3^{\circ}$ corte & 15 ago. 2019 \\
$4^{\circ}$ corte & 15 ago. 2020 \\
$5^{\circ}$ corte & 15 ago. 2021 \\
$6^{\circ}$ corte & 15 ago. 2022 \\
$7^{\circ}$ corte & 15 ago. 2023 \\
$8^{\circ}$ corte & 15 ago. 2024 \\
\hline
\end{tabular}

Fonte: Resultados originais da pesquisa

Tabela 10. Resultado da análise econômica dos indicadores econômicos Valor Presente Líquido para períodos não uniformes [XVPL] e Valor Presente Líquido Anualizado [VPLA], expressos em R\$, para os diferentes estágios da cultura

\begin{tabular}{|c|c|c|c|c|}
\hline \multirow{2}{*}{ Indicador econômico } & \multicolumn{4}{|c|}{ Valores de XVPL e VPLA } \\
\hline & $5^{\circ}$ corte & $6^{\circ}$ corte & $7^{\circ}$ corte & $8^{\circ}$ corte \\
\hline & - & - & 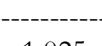 & \\
\hline XVPL & 412 & 803 & 1.025 & 1.104 \\
\hline VPLA & 95 & 159 & 178 & 171 \\
\hline
\end{tabular}

Fonte: Resultados originais da pesquisa

O maior resultado de VPLA foi encontrado no $7^{\circ}$ corte, indicando, que nesse momento foi alcançado o maior valor econômico para a lavoura. A partir de então os retornos econômicos se apresentaram com valores decrescentes, portanto por este método, os retornos econômicos passam a ser menores se for mantido o canavial para mais cortes. A orientação é para que se faça a substituição por um novo canavial que gerará melhores resultados econômicos após a colheita do $7^{\circ}$ corte.

Embora os resultados de VPLA nos cortes acima daquele estabelecido como ponto econômico de reforma ainda seja positivo, ele pode ser enganoso. Veiga Filho (2002) verificou que o ciclo da cana deveria ser finalizado no $5^{\circ}$ corte, podendo ir até o $6^{\circ}$ corte se houvesse condições favoráveis ao adiamento. Ele observou que ocorreram taxas decrescentes do VPLA do $5^{\circ}$ para $6^{\circ} \mathrm{e}$ $7^{\circ}$ cortes, porém houve um fluxo positivo de receita líquida, o que induziria o produtor a adiar a reforma pelo alto valor de investimento de uma nova cultura e dos custos financeiros. Por outro lado, com a reforma no $5^{\circ}$ corte, haveria a criação de um novo fluxo de renda líquida, maior que a anterior. A decisão de reforma neste caso seria direcionada por critério econômico e não apenas pela produtividade.

A situação foi semelhante neste trabalho, embora o ponto econômico tenha ocorrido no $7^{\circ}$ corte. Esse foi um grande orientador de que variando os fatores que compõem o fluxo de caixa dos projetos, outros 
resultados de VPLA poderão ser encontrados, portanto, uma análise mais criteriosa foi feita considerando alterações de todos os indicadores de produção estudados e analisando os seus efeitos em cada ciclo do canavial estabelecido para estudo, por meio da análise de sensibilidade.

\section{Analise de sensibilidade}

Os resultados encontrados na análise de sensibilidade e, os impactos dos indicadores nos resultados de VPLA, calculados, conforme a metodologia descrita, estão apresentados nas tabelas 11 a 17.

O efeito da variação da produtividade de primeiro corte pode ser visto na tabela 11, sendo necessário esclarecer que as produtividades de $2^{\circ}$ aos demais cortes seguiram as quebras de produtividades de um corte para outro, conforme estabelecido como premissa básica, já demonstrada anteriormente, para fins de verificação da aplicabilidade do instrumento de análise econômica.

Nota-se que foi preciso uma produtividade inicial de $1^{\circ}$ corte acima de $140 \mathrm{t} \mathrm{ha}^{-1}$ para que o ponto ótimo de reforma econômica fosse alterado de $7^{\circ}$ para o $6^{\circ}$ corte, nas condições desse trabalho, variando esse indicador, sem alterar as demais variáveis. Considerando esse fato, partindo de uma produtividade média de $1^{\circ}$ corte de 140 t ha ${ }^{-1}$ e obedecendo-se as regras estabelecidas de queda de produtividade apresentado como premissa na tabela 4, a produtividade no $6^{\circ}$ corte seria de $88 \mathrm{t} \mathrm{ha}^{-1}$, considerada alta para as médias nacionais, porém $\mathrm{O}$ resultado econômico deixa claro que houve maior retorno do investimento, com orientação para a reforma nesse estágio de corte, mesmo com produtividade agrícola mais alta que os padrões normais de indicação de reforma pelos critérios tradicionalmente utilizados.

Tabela 11. Resultados de Valor Presente Líquido Anualizado [VPLA], em R\$, em cada estágio da cultura, para variações da produtividade inicial de $1^{\circ}$ corte, em t ha ${ }^{-1}$

\begin{tabular}{|c|c|c|c|c|}
\hline \multirow{2}{*}{ Produtividade inicial para $1^{\circ}$ corte } & \multicolumn{4}{|c|}{ VPLA } \\
\hline & $5^{\circ}$ corte & $6^{\circ}$ corte & $7^{\circ}$ corte & $8^{\circ}$ corte \\
\hline ------- t ha-1 ----------- & \multicolumn{4}{|c|}{ 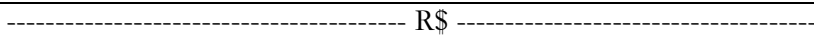 } \\
\hline 110 & -28 & 40 & 63 & 60 \\
\hline 114 & 95 & 159 & 178 & 171 \\
\hline 120 & 280 & 337 & 350 & 338 \\
\hline 130 & 589 & 635 & 637 & 616 \\
\hline 140 & 897 & 932 & 925 & 894 \\
\hline
\end{tabular}

Fonte: Resultados originais da pesquisa

Trabalho conduzido em canavial da região de Ribeirão Preto, São Paulo, ambiente A, considerado de alto potencial produtivo, partindo de produtividade inicial de 134,5 t ha ${ }^{-1}$, utilizando ferramenta de análise econômica semelhante, a reforma do canavial deveria se dar com produtividade de $84,4 \mathrm{t} \mathrm{ha}^{-1}$ obtida no $4^{\mathrm{o}}$ corte $^{1}$. Pode-se afirmar, portanto, que o potencial produtivo da lavoura altera o ponto econômico da reforma e leva o canavial a ser reformado com produtividades maiores daquelas que se têm estabelecido pelos padrões das principais empresas e técnicos que se baseiam na produtividade agrícola como referência de reforma.

A tabela 12 apresenta os resultados do VPLA, expresso em R \$, para os estágios da cultura estudados, considerando-se as variações da taxa real de juros anual, em $\%$.

Tabela 12. Resultado do Valor Presente Líquido Anualizado [VPLA], em R\$, em cada estágio da cultura, para as variações da taxa real de juros anual, em \%

\begin{tabular}{|c|c|c|c|c|}
\hline \multirow{2}{*}{ Taxa real de juros anual } & \multicolumn{4}{|c|}{ VPLA } \\
\hline & $5^{\circ}$ corte & $6^{\circ}$ corte & $7^{\circ}$ corte & $8^{\circ}$ corte \\
\hline --------- \% --------- & ---------- & ----------- & ---------- & ---------- \\
\hline 4 & 145 & 205 & 220 & 211 \\
\hline 6 & 53 & 119 & 141 & 138 \\
\hline 8 & -41 & 31 & 59 & 62 \\
\hline 10 & -137 & -59 & -25 & -17 \\
\hline 12 & -234 & -150 & -112 & -100 \\
\hline
\end{tabular}

Fonte: Resultados originais da pesquisa

Os melhores resultados de VPLA foram alcançados quando as taxas de juros foram mais baixas e, o $7^{\circ}$ corte foi aquele considerado o ponto econômico de reforma, porém, a uma taxa de $8 \%$ a.a., devido aos custos mais elevados de captação de recursos, o maior resultado de VPLA indicou a necessidade do adiamento da renovação

${ }^{1}$ Sacarelli, M.O. 2007. Utilização da margem de contribuição agrícola na determinação do número ótimo de cortes para manejo da cultura da cana-de-açúcar. 31 p.Trabalho de Conclusão de Curso (Agronomia) - Faculdade de Ciências Agrárias e Veterinárias, Universidade Estadual de São Paulo, Jaboticabal. 
do canavial para o $8^{\circ}$ corte e quando em condições de taxa de juros de 10 e $12 \%$ a.a., todos os projetos analisados tornaram-se inviáveis. Os dados referentes aos impactos das variações dos valores desse indicador, sugeridas pelo autor, estão apresentados na tabela 13 .

Tabela 13. Valor Presente Líquido Anualizado [VPLA], em R $\$$, em cada estágio da cultura, para variações do preço da tonelada de cana, em $\mathrm{R} \$ \mathrm{t}^{-1}$

\begin{tabular}{|c|c|c|c|c|}
\hline \multirow{2}{*}{ Preço da tonelada de cana } & \multicolumn{4}{|c|}{ VPLA } \\
\hline & $5^{\circ}$ corte & $6^{\circ}$ corte & $7^{\circ}$ corte & $8^{\circ}$ corte \\
\hline ------ R $\$ t^{-1}$ & --- & 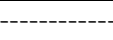 & -------- & ------- \\
\hline 60 & -351 & -272 & -238 & -231 \\
\hline 65 & 103 & 166 & 185 & 178 \\
\hline 70 & 556 & 603 & 607 & 587 \\
\hline 75 & 1.010 & 1.041 & 1.030 & 996 \\
\hline 80 & 1.463 & 1.478 & 1.452 & 1.405 \\
\hline
\end{tabular}

Fonte: Resultados originais da pesquisa

O aumento do preço da tonelada da cana implicou em aumentos dos resultados de VPLA. Aos preços praticados, descritos na tabela 13 , o ponto ótimo econômico para reforma continuou sendo no $7^{\circ}$ corte, exceto nas condições de melhora nos preços por $t$, por exemplo, de $\mathrm{R} \$ 80,00$, onde o melhor resultado econômico aconteceu no $6^{\circ}$ corte e com orientação da reforma para esse estágio.

Os resultados de VPLA para variações da taxa de arrendamento, mantendo-se as demais condições do trabalho inalteradas (ceteris paribus), estão apresentados na tabela 14.

Tabela 14. Valor Presente Líquido Anualizado [VPLA], em cada estágio da cultura, considerando-se as variações da taxa de arrendamento, em $\mathrm{t} \mathrm{ha}^{-1}$

\begin{tabular}{|c|c|c|c|c|}
\hline \multirow{3}{*}{ Taxa de arrendamento } & \multicolumn{4}{|c|}{ Valores de VPLA } \\
\hline & \multicolumn{4}{|c|}{ Estágio da cultura } \\
\hline & $5^{\circ}$ corte & $6^{\circ}$ corte & $7^{\circ}$ corte & $8^{\circ}$ corte \\
\hline ------- t ha'-1 ------ & ----------- & ------ & ----------- & ---------- \\
\hline 18 & 97 & 160 & 179 & 173 \\
\hline 20 & -17 & 46 & 65 & 59 \\
\hline 22 & -131 & -67 & -48 & -55 \\
\hline 24 & -245 & -181 & -163 & -169 \\
\hline 26 & -358 & -295 & -276 & -282 \\
\hline
\end{tabular}

Fonte: Resultados originais da pesquisa

O melhor resultado econômico foi obtido com a taxa de arrendamento de $18 \mathrm{t} \mathrm{ha}^{-1}$, indicando a reforma no $7^{\circ}$ corte. Taxas maiores de arrendamento, acima de $22 \mathrm{t}$ ha ${ }^{1}$, tornaram inviável o projeto e nesse caso a orientação para o caso é de que seja trocado por outros de maior rentabilidade. A tabela 15 apresenta os resultados de VPLA (R\$) considerando os impactos causados pelas variações dos valores do custo desembolsável de colheita $\left(\mathrm{R} \$ \mathrm{t}^{-1}\right)$ no FCL para os estágios da cultura estudados.

Tabela 15. Resultados de Valor Presente Líquido Anualizado [VPLA] em cada estágio da cultura, para variações do custo desembolsável de colheita, em $\mathrm{R} \$ \mathrm{t}^{-1}$

\begin{tabular}{ccccc}
\hline \multirow{2}{*}{ Custo desembolsável de colheita } & \multicolumn{3}{c}{ VPLA } \\
\cline { 2 - 5 } & $5^{\text {o }}$ corte & $6^{\text {o }}$ corte & $7^{\circ}$ corte & $8^{\circ}$ corte \\
\hline 18 & -197 & 836 & 832 & 804 \\
20 & 612 & 657 & 659 & 637 \\
22 & 426 & 477 & 486 & 469 \\
24 & 240 & 298 & 313 & 302 \\
26 & 54 & 119 & 140 & 135 \\
\hline
\end{tabular}

Fonte: Resultados originais da pesquisa

Os resultados de VPLA foram maiores para os menores custos desembolsáveis de colheita, e quando esses custos foram de $\mathrm{R} \$ 18,00$ a tonelada, pode-se estabelecer um estágio menor de reforma por apresentar maior rentabilidade econômica no $6^{\circ}$ corte, mas ainda $\mathrm{o}$ ponto econômico de reforma, na maioria das faixas de custos analisados, ocorreu no $7^{\circ}$ corte e, portanto, foi o mais indicado para a reforma. 
A Tabela 16 apresenta os resultados do VPLA $(\mathrm{R} \$)$ com as variações do custo desembolsável na formação da lavoura $\left(\mathrm{R} \$ \mathrm{ha}^{-1}\right)$.

Tabela 16. Valor Presente Líquido Anualizado [VPLA] para variações do custo da formação do canavial, em $\mathrm{R} \$$ ha-1

\begin{tabular}{ccccc}
\hline \multirow{2}{*}{ Desembolso na formação do canavial } & \multicolumn{3}{c}{ Valores de VPLA } \\
\cline { 2 - 5 } & $5^{\circ}$ corte & $6^{\circ}$ corte & $7^{\circ}$ corte & $8^{\circ}$ corte \\
\hline 5.000 & -165 & 474 & 454 & 419 \\
5.500 & 349 & 375 & 367 & 341 \\
6.000 & 233 & 276 & 174 & 264 \\
6.500 & 117 & 79 & 107 & 186 \\
7.000 & 2 & 79 & 108 \\
\hline
\end{tabular}

Fonte: Resultados originais da pesquisa

Os resultados mostram a possibilidade de alternância da decisão da reforma quando se tem variações nos custos. Assim, custos menores indicam a possibilidade de reforma de canavial com seis cortes e custos maiores da formação de lavoura, obrigatoriamente indicaram a necessidade de adiar a reforma para o $7^{\circ}$ e $8^{\circ}$ corte.
O $7^{\circ}$ corte apareceu com os melhores pontos econômicos para reforma quando houve a variação no custo desembolsável de formação do canavial.

A tabela 17 apresenta o VPLA, em R \$, considerando as variações nos custos desembolsáveis de tratos culturais da cana-soca.

Tabela 17. Valor Presente Líquido Anualizado [VPLA], em R\$, com a variação dos custos desembolsáveis de tratos culturais de cana-soca, em $\mathrm{R} \$ \mathrm{ha}^{-1}$

\begin{tabular}{|c|c|c|c|c|}
\hline \multirow{2}{*}{ Custo desembolsável de tratos culturais de cana-soca } & \multicolumn{4}{|c|}{ VPLA } \\
\hline & $5^{\circ}$ corte & $6^{\circ}$ corte & $7^{\circ}$ corte & $8^{\circ}$ corte \\
\hline ------- R\$ ha-1 -------- & -------- & 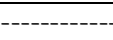 & 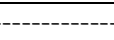 & 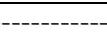 \\
\hline 1.000 & 160 & 226 & 247 & 242 \\
\hline 1.100 & 80 & 143 & 161 & 155 \\
\hline 1.200 & 0 & 59 & 75 & 67 \\
\hline 1.300 & -80 & -24 & -11 & -21 \\
\hline 1.400 & -161 & -108 & -97 & -108 \\
\hline
\end{tabular}

Fonte: Resultados originais da pesquisa

Os maiores resultados de VPLA apresentados na tabela 17 indicaram o $7^{\circ}$ corte como ponto econômico para reforma de um canavial, e são inversamente proporcionais aos custos desembolsáveis de tratamento da cana-soca.

\section{Resultados das interações dos indicadores}

Foi possível observar que dependendo das variáveis estudadas, o ponto econômico, orientador de reforma, é alterado e, portanto, os resultados não podem ser tratados isoladamente. Em algumas situações, esse ponto econômico foi alcançado no $6^{\circ}$ ou no $8^{\circ}$ corte, apesar do predomínio do $7^{\circ}$ corte como o estágio mais indicado para reforma. Isso demonstra a dinamicidade do recurso de uso do indicador econômico apresentado e que a sua aplicação pode ter um impacto muito expressivo no setor sucroenergético.
Ainda, foi possível analisar os resultados do VPLA causados pelos impactos das variações dos indicadores de produção, quando estudados por meio de suas interações conjuntamente. Algumas das principais interações entre indicadores e seus resultados de análise econômica estão descritos a seguir.

\section{Interação entre Taxa real de juros e Preço da tonelada de cana}

A tabela 18 apresenta os efeitos da variação da taxa real de juros e preço da tonelada de cana conjuntamente. Os resultados mostraram o VPLA quando os indicadores foram cruzados entre si e analisados pelo método adotado. Nota-se que, dependendo das condições, pode-se ter mudança na tomada de decisão de reformar o canavial. Preços da tonelada de cana de $\mathrm{R} \$ 60,00$ tornaram todos os projetos inviáveis quando consideradas as taxas de juros analisadas. 
Tabela 18. Valor Presente Líquido Anualizado [VPLA], em reais, com a interação, conjuntamente, das variações da Taxa real de juros e Preço da tonelada de cana, em $\mathrm{R} \$ \mathrm{t}^{-1}$

\begin{tabular}{|c|c|c|c|c|c|c|}
\hline \multirow{3}{*}{$\begin{array}{l}\text { Número de } \\
\text { cortes }\end{array}$} & \multirow{3}{*}{$\begin{array}{c}\text { Preço da tonelada } \\
\text { de cana }\end{array}$} & \multicolumn{5}{|c|}{ Valores de VPLA } \\
\hline & & \multicolumn{5}{|c|}{ Taxa real de juros anual } \\
\hline & & $4 \%$ & $6 \%$ & $8 \%$ & $10 \%$ & $12 \%$ \\
\hline \multirow{6}{*}{5} & ------- R\$ t-1 --------- & & --------" & -- $\mathrm{R} \$-$ & -------- & \\
\hline & 60 & -302 & -392 & -484 & -578 & -673 \\
\hline & 65 & 153 & 60 & -34 & -129 & -226 \\
\hline & 70 & 608 & 513 & 417 & 319 & 220 \\
\hline & 75 & 1.062 & 966 & 868 & 768 & 667 \\
\hline & 80 & 1.517 & 1.418 & 1.318 & 1.217 & 1.114 \\
\hline \multirow{5}{*}{6} & 60 & -226 & -310 & -397 & -486 & -576 \\
\hline & 65 & 212 & 127 & 39 & -51 & -143 \\
\hline & 70 & 650 & 563 & 474 & 383 & 290 \\
\hline & 75 & 1.089 & 1.000 & 910 & 817 & 723 \\
\hline & 80 & 1.527 & 1.437 & 1.345 & 1.251 & 1.156 \\
\hline \multirow{5}{*}{7} & 60 & -195 & -274 & -356 & -439 & -526 \\
\hline & 65 & 227 & 148 & 66 & -18 & -105 \\
\hline & 70 & 650 & 570 & 488 & 403 & 315 \\
\hline & 75 & 1.073 & 993 & 910 & 824 & 736 \\
\hline & 80 & 1.496 & 1.415 & 1.331 & 1.245 & 1.156 \\
\hline \multirow{5}{*}{8} & 60 & -191 & -264 & -341 & -420 & -502 \\
\hline & 65 & 217 & 145 & 68 & -11 & -93 \\
\hline & 70 & 626 & 553 & 478 & 399 & 316 \\
\hline & 75 & 1.035 & 962 & 887 & 808 & 726 \\
\hline & 80 & 1.444 & 1.371 & 1.296 & 1.217 & 1.135 \\
\hline
\end{tabular}

Fonte: Resultados originais da pesquisa

O preço da tonelada de cana em $\mathrm{R} \$ 80,00$ indicou o ponto econômico sendo atingido, em qualquer uma das taxas estudadas, no $6^{\circ}$ corte. À medida que os preços foram caindo e as taxas aumentando, o ponto econômico de reforma indicou o $7^{\circ}$ corte como melhor alternativa.

\section{Interação entre Custo desembolsável dos tratos de} cana-soca e Produtividade agrícola

Os efeitos conjuntos das variações do custo desembolsável dos tratos da cana-soca foram cruzados com as variações da produtividade do canavial, com a intenção de analisar os resultados de VPLA que estão apresentados na tabela 19 . Os resultados foram maiores quando teve aumento de produtividade de cana no $1^{\circ}$ corte e redução dos custos desembolsáveis de tratos culturais de cana-soca. Considerou-se a produtividade do $1^{\circ}$ corte, para que fossem aplicadas as quebras de produtividades, para fins de cálculo do FCL. Essas quebras foram estabelecidas nas premissas básicas, citadas na tabela 4, determinando uma quebra de produtividade de $1^{\circ}$ corte para o $2^{\circ}$ na ordem de $12 \%$ e nos demais cortes quebra de $8 \%$. O mesmo critério já foi adotado para a análise dos dados apresentados na tabela 11 e também será utilizado nas tabelas 20 e 21 .
Os melhores resultados mostraram que, em casos de altas produtividades com o cruzamento de todos os valores relacionados aos custos de tratos culturais de cana-soca, os pontos econômicos máximos apareceram no $6^{\circ}$ corte, porém, nos casos das produtividades menores, dentro da faixa estabelecida na análise, na maioria dos resultados houve a indicação de que o momento ótimo de reforma deveria ser no $7^{\circ}$ corte. Para os casos de impacto da redução de despesas com tratos culturais, na produtividade causando a sua queda, comum no setor em época de crise, pode-se concluir que os melhores resultados econômicos apareceram no $7^{\circ}$ corte.

\section{Interação entre Custo desembolsável na formação} da lavoura e Produtividade agrícola

O VPLA obtido pelas variações desses indicadores está apresentado na tabela 20. Notou-se que custos maiores de formação da lavoura direcionaram a reforma para o $7^{\circ}$ corte, mas na maioria dos casos o cruzamento desses indicadores concentrou a reforma no $6^{\circ}$ corte, com a possibilidade até de antecipar para o $5^{\circ}$ corte nos casos de baixos valores desembolsáveis na formação do canavial e de produtividades agrícolas esperadas atingindo valores superiores a $130 \mathrm{t} \mathrm{ha}^{-1}$. 
Tabela 19. Resultado de Valor Presente Líquido Anualizado [VPLA], em R\$, para interação conjuntamente da variação do custo desembolsável de tratos culturais de cana-soca, em $\mathrm{R} \$ \mathrm{ha}^{-1}$ e da variação da produtividade de cana do $1^{\circ}$ corte, em $\mathrm{t}$ ha $^{-1}$, tomando-se como base dados de um canavial da região tradicional Centro-Sul do Brasil

\begin{tabular}{|c|c|c|c|c|c|c|}
\hline \multirow{3}{*}{ Número de cortes } & \multirow{3}{*}{$\begin{array}{l}\text { Produtividade de cana do } 1^{\circ} \\
\text { corte }\end{array}$} & \multicolumn{5}{|c|}{ VPLA } \\
\hline & & \multicolumn{5}{|c|}{ Custo de tratos culturais de cana-soca $\left(\mathrm{R} \$ \text { ha }^{-1}\right)^{*}$} \\
\hline & & 1.000 & 1.100 & 1.200 & 1.300 & 1.400 \\
\hline \multirow{6}{*}{5} & ---------- t ha' ${ }^{-1}$---------- & $-\overline{-----}$ & - & $\mathrm{R} \$-$ & ------ & ---- \\
\hline & 110 & 37 & -43 & -124 & -204 & -284 \\
\hline & 114 & 160 & 80 & 0 & -80 & -161 \\
\hline & 120 & 345 & 265 & 185 & 105 & 24 \\
\hline & 130 & 654 & 573 & 493 & 413 & 333 \\
\hline & 140 & 962 & 882 & 802 & 721 & 641 \\
\hline \multirow{5}{*}{6} & 110 & 107 & 24 & -60 & -143 & -227 \\
\hline & 114 & 226 & 143 & 59 & -24 & -108 \\
\hline & 120 & 405 & 321 & 238 & 154 & 71 \\
\hline & 130 & 702 & 619 & 535 & 451 & 368 \\
\hline & 140 & 1.000 & 916 & 832 & 749 & 665 \\
\hline \multirow{5}{*}{7} & 110 & 132 & 46 & -40 & -126 & -211 \\
\hline & 114 & 247 & 161 & 75 & -11 & -97 \\
\hline & 120 & 420 & 334 & 248 & 162 & 76 \\
\hline & 130 & 707 & 621 & 535 & 449 & 363 \\
\hline & 140 & 994 & 908 & 822 & 736 & 651 \\
\hline \multirow{5}{*}{8} & 110 & 131 & 43 & -44 & -132 & -220 \\
\hline & 114 & 242 & 155 & 67 & -21 & -108 \\
\hline & 120 & 409 & 321 & 234 & 146 & 58 \\
\hline & 130 & 687 & 599 & 512 & 424 & 336 \\
\hline & 140 & 965 & 877 & 790 & 702 & 614 \\
\hline
\end{tabular}

* custo desembolsável

Fonte: Resultados originais da pesquisa

Tabela 20. Valor Presente Líquido Anualizado [VPLA] para interação, conjuntamente, da variação do Custo desembolsável de Formação da lavoura de cana-de-açúcar, em $\mathrm{R} \$ \mathrm{ha}^{-1}$, e da variação da produtividade do $1^{\circ}$ corte, em $\mathrm{t}$ ha ${ }^{-1}$

\begin{tabular}{|c|c|c|c|c|c|c|}
\hline \multirow{3}{*}{$\begin{array}{l}\text { Número de } \\
\text { cortes }\end{array}$} & \multirow{3}{*}{$\begin{array}{l}\text { Produtividade de cana } \\
\text { do } 1^{\circ} \text { corte }\end{array}$} & \multicolumn{5}{|c|}{ Valores de VPLA } \\
\hline & & \multicolumn{5}{|c|}{ Desembolso na formação da lavoura de cana $\left(\mathrm{R} \$\right.$ ha $\left.^{-1}\right)$} \\
\hline & & 5.000 & 5.500 & 6.000 & 6.500 & 7.000 \\
\hline & ---------- $\mathrm{t} \mathrm{ha}^{-1}$--------- & \multicolumn{5}{|c|}{ 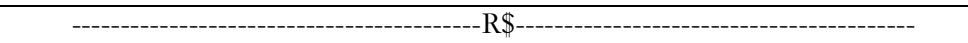 } \\
\hline \multirow{5}{*}{5} & 110 & 341 & 226 & 110 & -6 & -122 \\
\hline & 114 & 465 & 349 & 233 & 117 & 2 \\
\hline & 120 & 650 & 534 & 418 & 302 & 187 \\
\hline & 130 & 958 & 842 & 726 & 611 & 495 \\
\hline & 140 & 1.266 & 1.151 & 1.035 & 919 & 803 \\
\hline \multirow{5}{*}{6} & 110 & 355 & 256 & 157 & 58 & -40 \\
\hline & 114 & 476 & 375 & 276 & 177 & 79 \\
\hline & 120 & 652 & 553 & 455 & 356 & 257 \\
\hline & 130 & 950 & 851 & 752 & 653 & 554 \\
\hline & 140 & 1.247 & 1.148 & 1.049 & 951 & 852 \\
\hline \multirow{5}{*}{7} & 110 & 339 & 253 & 166 & 79 & -8 \\
\hline & 114 & 454 & 367 & 281 & 194 & 107 \\
\hline & 120 & 627 & 540 & 453 & 367 & 280 \\
\hline & 130 & 914 & 827 & 741 & 654 & 567 \\
\hline & 140 & 1.201 & 1.115 & 1028 & 941 & 854 \\
\hline \multirow{5}{*}{8} & 110 & 308 & 230 & 153 & 75 & -3 \\
\hline & 114 & 419 & 341 & 264 & 186 & 108 \\
\hline & 120 & 586 & 508 & 431 & 353 & 275 \\
\hline & 130 & 864 & 786 & 709 & 631 & 553 \\
\hline & 140 & 1.142 & 1.064 & 987 & 909 & 831 \\
\hline
\end{tabular}

Fonte: Resultados originais da pesquisa 


\section{Interação entre Preço da cana e Produtividade agrícola}

A tabela 21 apresenta o VPLA para as correspondentes variações dos indicadores técnicos preço da cana e a produtividade agrícola do canavial.

Os resultados mostraram que, nos casos de baixos preços da cana, o ponto de reforma apontou para o $7^{\circ}$ corte como melhor opção. À medida que os preços foram aumentando, o ponto econômico de reforma migrou para o $6^{\circ}$ corte, com indicativo de poder chegar ao $5^{\circ}$ corte nos casos de preço mais alto e produtividade de primeiro corte também alta (Tabela 21).

Tabela 21. Valor Presente Líquido Anualizado [VPLA], em R\$, para interação, conjuntamente, da variação de Preço da cana, em $\mathrm{R} \$ \mathrm{t}^{-1}$, e da variação da Produtividade da cana em seu $1^{\circ}$ corte, em tha ${ }^{-1}$

\begin{tabular}{|c|c|c|c|c|c|c|}
\hline \multirow{3}{*}{$\begin{array}{l}\text { Número de } \\
\text { cortes }\end{array}$} & \multirow{3}{*}{$\begin{array}{c}\text { Produtividade de cana } \\
\text { do } 1^{\circ} \text { corte }\end{array}$} & \multicolumn{5}{|c|}{ VPLA } \\
\hline & & \multicolumn{5}{|c|}{ Preço da cana $\left(\mathrm{R} \$ \mathrm{t}^{-1}\right)$} \\
\hline & & 60 & 65 & 70 & 75 & 80 \\
\hline \multirow{6}{*}{5} & ------ $\mathrm{t} \mathrm{ha}^{-1}$------- & & - & $--\mathrm{R} \$--$ & - & \\
\hline & 110 & -458 & -21 & 417 & 854 & 1.292 \\
\hline & 114 & -351 & 103 & 556 & 1.010 & 1.463 \\
\hline & 120 & -189 & 288 & 766 & 1.243 & 1.720 \\
\hline & 130 & 80 & 597 & 1.114 & 1.632 & 2.149 \\
\hline & 140 & 349 & 906 & 1.463 & 2.020 & 2.577 \\
\hline \multirow{5}{*}{6} & 110 & -375 & 47 & 469 & 891 & 1.313 \\
\hline & 114 & -272 & 166 & 603 & 1.041 & 1.478 \\
\hline & 120 & -116 & 345 & 805 & 1.266 & 1.726 \\
\hline & 130 & 144 & 643 & 1.142 & 1.640 & 2.139 \\
\hline & 140 & 404 & 941 & 1.478 & 2.015 & 2.552 \\
\hline \multirow{5}{*}{7} & 110 & -338 & 69 & 477 & 885 & 1.293 \\
\hline & 114 & -238 & 185 & 607 & 1.030 & 1.452 \\
\hline & 120 & -87 & 357 & 802 & 1.247 & 1.692 \\
\hline & 130 & 163 & 645 & 1.127 & 1.609 & 2.091 \\
\hline & 140 & 414 & 933 & 1.452 & 1.971 & 2.490 \\
\hline \multirow{5}{*}{8} & 110 & -328 & 67 & 461 & 856 & 1.250 \\
\hline & 114 & -231 & 178 & 587 & 996 & 1.405 \\
\hline & 120 & -85 & 345 & 776 & 1.206 & 1.636 \\
\hline & 130 & 158 & 624 & 1.090 & 1.556 & 2.023 \\
\hline & 140 & 400 & 902 & 1.405 & 1.907 & 2.409 \\
\hline
\end{tabular}

Fonte: Resultados originais da pesquisa

Quando os preços foram os mais baixos, para que os projetos se tornassem viáveis economicamente, foram necessárias produtividades maiores de $1^{\circ}$ corte.

Tomando por base a tabela $21 \mathrm{e}$, considerando uma situação na qual os preços da cana atingem cerca de $\mathrm{R} \$ 70,00$ a tonelada e a produtividade esperada para o $1^{\circ}$ corte de $114 \mathrm{t} \mathrm{ha}^{-1}$, levando-se em consideração as premissas adotadas, o melhor resultado de VPLA encontra-se no $7^{\circ}$ corte, podendo ser considerado como o momento de maior valor econômico alcançado e, portanto, devendo ser orientado para a reforma, de acordo com os conceitos propostos pelo trabalho.

\section{Conclusões}

O ponto econômico de reforma do canavial, para os maiores resultados de VPLA, sem considerar as alterações nas premissas básicas, com base nos dados de um canavial da região tradicional do Centro-Sul do Brasil, acontece no $7^{\circ}$ corte, com produtividade do canavial de $66 \mathrm{t} \mathrm{ha}^{-1}$.
Os indicadores de produção: produtividade agrícola inicial $\left(\mathrm{t} \mathrm{ha} \mathrm{h}^{-1}\right)$ e preço da tonelada da cana $\left(\mathrm{R} \$ \mathrm{t}^{-1}\right)$, ao serem submetidos à análise de sensibilidade, causam impactos diretamente proporcionais nos resultados de VPLA e provocam a tomada de decisão de reforma de $7^{\circ}$ para o $6^{\circ}$ corte quando a estes são aplicados valores maiores na análise, indicando que a expectativa de melhores resultados proporciona a possibilidade de antecipação de reforma dos canaviais em função das receitas geradas pela maior produção e pelos maiores retornos com o preço da tonelada de cana, porém os indicadores: taxa real de juros (\%), custo desembolsável de colheita $\left(\mathrm{R} \$ \mathrm{t}^{-1}\right)$ e custo desembolsável na formação da lavoura $\left(\mathrm{R} \$ \mathrm{ha}^{-1}\right)$, causam efeitos inversos nos resultados do VPLA quando seus valores são crescentes na análise.

Os indicadores: taxa de arrendamento $\left(\mathrm{t} \mathrm{ha}^{-1}\right)$ e custo desembolsável de tratos culturais $\left(\mathrm{R} \$ \mathrm{ha}^{-1}\right)$ também apresentam a mesma tendência de resultados de VPLA menores, à medida que a sua variação é crescente, mas se mantêm com a indicação do $7^{\circ}$ corte como melhor opção para reforma. Alguns resultados econômicos apontam 
para a indicação de decisão de reforma de canavial em estágios de corte com produtividades superiores àquelas que normalmente se tem adotado no setor, por isso precisam ser analisados com muito critério pelos tomadores de decisão.

Por estarem muito interligados, alguns indicadores de produção precisam ser analisados conjuntamente e, seus resultados, podem ser facilmente observados quando colocados em matrizes com o cruzamento desses dados. Os resultados mostram que a combinação de dois indicadores provocam alterações significativas na tomada de decisão.

Verifica-se a possibilidade de tomada de decisão de reforma do $6^{\circ}$ para o $7^{\circ}$ corte, à medida que se tem, conjuntamente, aumento na taxa de juros (\%) e redução do preço da tonelada de cana $\left(\mathrm{R} \$ \mathrm{t}^{-1}\right)$. O mesmo acontece quando é aplicada redução no custo desembolsável de tratos culturais $\left(\mathrm{R} \$ \mathrm{ha}^{-1}\right)$ e queda na expectativa de produtividade inicial do canavial $\left(\mathrm{t} \mathrm{ha}^{-1}\right)$.

O custo desembolsável para a formação da lavoura $\left(\mathrm{R} \$ \mathrm{ha}^{-1}\right)$ quando analisado conjuntamente com a expectativa inicial de produtividade $\left(\mathrm{t} \mathrm{ha}^{-1}\right)$ demonstra ter forte impacto no VPLA, indicando que seu valor crescente pode alterar a tomada de decisão de reforma do canavial do $5^{\circ}$ para o $6^{\circ}$ corte, podendo até ser no $7^{\circ}$ corte, visto que a necessidade de gastos maiores na formação da lavoura causam impedimentos financeiros na reforma do canavial.

Os resultados demonstram que o aumento dos valores recebidos pela tonelada da cana $\left(R \$ t^{-1}\right)$, quando comparado conjuntamente com a expectativa de produtividade inicial da lavoura $\left(\mathrm{t} \mathrm{ha}^{-1}\right)$, provoca a redução da decisão da reforma de $7^{\circ}$ corte para o $6^{\circ}$ corte, podendo chegar até a ocorrer no $5^{\circ}$ corte.

A análise econômica feita por meio do VPLA é uma opção muito adequada e que deve ser extrapolada, podendo ser indicada aos gestores como alternativa nas tomadas de decisão de reforma dos canaviais.

\section{Referências}

Assaf Neto, A. 1992. Os métodos quantitativos de análise de investimentos. Caderno de Estudos 6: 1-16. Banco Central do Brasil [BACEN]. 2016. Taxa SELIC

- Meta. Disponível em < https://www.bcb.gov.br/ptbr/\#!/home>. Acesso em: 20 out. 2016.
Borba, M.M.Z.; Bazzo, A.M. 2009. Estudo econômico do ciclo produtivo da cana-de-açúcar para reforma de canavial, em área de fornecedor do Estado de São Paulo. In: $47^{\circ}$ Congresso da SOBER, 2009, Porto Alegre. Anais do $47^{\circ}$ Congresso da SOBER.

Disponível em:

<http://www.sober.org.br/palestra/13/1169.pdf $>$. Acesso em: 21 set. 2016.

Cruz, M.R.O; Magalhães, M.M. 2013. Rotação de cultura e efeito sobre os custos na reforma de canavial na região da Alta Paulista. Fórum Ambiental da Alta Paulista 9: 92-109

Kuhnen, O.L.; Bauer, U.R. Matemática financeira aplicada e análise de investimentos. Atlas. São Paulo, São Paulo, Brasil.

Marim, W.C. Análise de alternativas de investimento: uma abordagem financeira. Atlas, São Paulo, São Paulo, Brasil.

Mello, R.C. 2005. Influência do formato e velocidade da lâmina nas forças de corte para cana-de-açúcar. Acta Scientiarum 27: 661-665.

Programa de Educação Continuada em Economia e Gestão de Empresas, Departamento de Economia, Administração e Sociologia. [PECEGE]. 2015. Custos de produção de cana-de-açúcar, açúcar, etanol e bioeletricidade no Brasil: fechamento da safra 2014/2015 e acompanhamento da safra 2015/2016. Universidade de São Paulo, Piracicaba, São Paulo, Brasil.

Samanez, C.P. 2010. Matemática financeira: aplicações à análise e investimentos. 5ed. Pearson Education, São Paulo, São Paulo, Brasil.

Souza, A. 2004. Decisões financeiras e análise de investimentos: fundamentos, técnicas e aplicações. 5ed. Atlas, São Paulo, São Paulo, Brasil.

Souza, A.; Clemente, A. 2004. Decisões financeiras e análise de investimento. Atlas, São Paulo, São Paulo, Brasil.

Veiga Filho, A.A. 2002. Quando reformar um canavial? Disponível em: <http://www.iea.sp.gov.br/out/ver'Texto.php?codTe xto $=110>$ Acesso em: 13 set. 2016. 